\title{
UNMANNED SYSTEMS
}

http://www.worldscientific.com/worldscinet/us

\section{Author Index \\ Volume 1 (2013)}

Ang, K. Z. Y., see Lin, F.

Beard, R. W., see Niedfeldt, P. C.

Brazinskas, M., see Prior, S. D.

Cai, G., see Lin, F.

Carroll, B. T., see Niedfeldt, P. C.

Chawla, C., see Raja, R. G.

Chen, B. M., see Lin, F.

Cui, J., see Lin, F.

de Silva, C. W., see Lang, H.

Dobrokhodov, V., see Keller, J.

Doherty, P., Heintz, F. and Kvarnström, J., High-Level Mission Specification and Planning for Collaborative

Unmanned Aircraft Systems Using Delegation

Dong, M., see Lin, F.

Dong, X., see Lin, F.

En Chao, J. J., Extractor $X-$ Autonomous Tilt Rotor UAV

Erbil, M. A., see Prior, S. D.

Gallier, J., see Keller, J.

Heintz, F., see Doherty, P.

How, J. P., see Kopeikin, A. N.

Howard, J. A., see Niedfeldt, P. C.

$\mathrm{Hu}, \mathrm{J.}, \mathrm{Xu}, \mathrm{J}$. and Xie, L., Cooperative Search and Exploration in Robotic Networks

Jackson, K., Li, J., Timmons, E. and Wallace, J., icaruslabs: An Adventure in Crowdsourcing

Johnson, L. B., see Kopeikin, A. N.

Jones, K., see Keller, J.

Kaminer, I., see Keller, J.

Kang, K. and Prasad, J. V. R., Development and Flight Test Evaluations of an Autonomous Obstacle Avoidance

System for a Rotary-Wing UAV

Keller, J., Thakur, D., Dobrokhodov, V., Jones, K., Pivtoraiko, M., Gallier, J., Kaminer, I. and Kumar, V.,

A Computationally Efficient Approach to Trajectory Management for Coordinated Aerial Surveillance

Khan, M. T., see Lang, H.

Kopeikin, A. N., Ponda, S. S., Johnson, L. B. and How, J. P., Dynamic Mission Planning for Communication

Control in Multiple Unmanned Aircraft Teams

Kumar, K. S. and Rasheed, A. M., Development of Rotary Wing Mini UAS for Civilian Applications

Kumar, V., see Keller, J.

Kvarnström, J., see Doherty, P.

Lang, H., Khan, M. T., Tan, K.-K. and de Silva, C. W., Developments in Visual Servoing for Mobile Manipulation

Lee, T. H., see Lin, F.

Li, J., see Jackson, K.

Li, K., see Lin, F.

Lin, F., Ang, K. Z. Y., Wang, F., Chen, B. M., Lee, T. H., Yang, B., Dong, M., Dong, X., Cui, J., Phang, S. K., Wang,

B., Luo, D., Peng, K., Cai, G., Zhao, S., Yin, M. and Li, K., Development of an Unmanned Coaxial Rotorcraft

for the DARPA UAVForge Challenge
1 (2013) 211-245

1 (2013) 277-296

1 (2013) 165-175

1 (2013) 211-245

1 (2013) 277-296

1 (2013) 259-275

1 (2013) 211-245

1 (2013) 211-245

1 (2013) 143-162

1 (2013) 59-74

1 (2013) 75-119

1 (2013) 211-245

1 (2013) 211-245

1 (2013) 177-198

1 (2013) 165-175

1 (2013) 59-74

1 (2013) 75-119

1 (2013) 41-58

1 (2013) 277-296

1 (2013) 121-142

1 (2013) 199-209

1 (2013) 41-58

1 (2013) 59-74

1 (2013) 59-74

1 (2013) 3-19

1 (2013) 59-74

1 (2013) 143-162

1 (2013) 41-58

1 (2013) 247-258

1 (2013) 59-74

1 (2013) 75-119

1 (2013) 143-162

1 (2013) 211-245

1 (2013) 199-209

1 (2013) 211-245

1 (2013) 211-245 
Luo, D., see Lin, F.

Mielniczek, W. P., see Prior, S. D.

Morse, B. S., see Niedfeldt, P. C.

Murray, R. M., see Wongpiromsarn, T.

Niedfeldt, P. C., Carroll, B. T., Howard, J. A., Beard, R. W., Morse, B. S. and Pledgie, S., Enhanced Uas Surveillance Using a Video Utility Metric

Padhi, R., see Raja, R. G.

Peng, K., see Lin, F.

Phang, S. K., see Lin, F.

Pivtoraiko, M., see Keller, J.

Pledgie, S., see Niedfeldt, P. C.

Ponda, S. S., see Kopeikin, A. N.

Prasad, J. V. R., see Kang, K.

Prior, S. D., Shen, S.-T., Erbil, M. A., Brazinskas, M. and Mielniczek, W. P., Winning the DARPA Uavforge Challenge 2012- Team HALO

Raja, R. G., Chawla, C. and Padhi, R., Dynamic Inversion-Based Nonlinear Aiming Point Guidance of Unmanned Aerial Vehicles for Reactive Obstacle Avoidance

Rasheed, A. M., see Kumar, K. S.

Shen, S.-T., see Prior, S. D.

Tan, K.-K., see Lang, H.

Thakur, D., see Keller, J.

Timmons, E., see Jackson, K.

Topcu, U., see Wongpiromsarn, T.

Wallace, J., see Jackson, K.

Wang, B., see Lin, F.

Wang, F., see Lin, F.

Wongpiromsarn, T., Topcu, U. and Murray, R. M., Synthesis of Control Protocols for Autonomous Systems

Xie, L., see $\mathrm{Hu}, \mathrm{J}$.

$\mathrm{Xu}, \mathrm{J}$. , see $\mathrm{Hu}, \mathrm{J}$.

Yang, B., see Lin, F.

Yin, M., see Lin, F.

Zhao, S., see Lin, F.

1 (2013) 211-245

1 (2013) 165-175

1 (2013) 277-296

1 (2013) 21-39

1 (2013) 277-296

1 (2013) 259-275

1 (2013) 211-245

1 (2013) 211-245

1 (2013) 59-74

1 (2013) 277-296

1 (2013) 41-58

1 (2013) 3-19

1 (2013) 165-175

1 (2013) 259-275

1 (2013) 247-258

1 (2013) 165-175

1 (2013) 143-162

1 (2013) 59-74

1 (2013) 199-209

1 (2013) 21-39

1 (2013) 199-209

1 (2013) 211-245

1 (2013) 211-245

1 (2013) 21-39

1 (2013) 121-142

1 (2013) 121-142

1 (2013) 211-245

1 (2013) 211-245

1 (2013) 211-245 